\title{
Large $\mathrm{D} / \mathrm{H}$ variations in bacterial lipids reflect central metabolic pathways
}

\author{
Xinning Zhang ${ }^{a}$, Aimee L. Gillespie ${ }^{b}$, and Alex L. Sessions ${ }^{a, b, 1}$ \\ aEnvironmental Science and Engineering Program and bDivision of Geological and Planetary Sciences, California Institute of Technology, \\ Pasadena, CA 91125 \\ This Feature Article is part of a series identified by the Editorial Board as reporting findings of exceptional significance.
}

Edited by John M. Hayes, Woods Hole Oceanographic Institution, Woods Hole, MA, and approved May 29, 2009 (received for review March 19, 2009 )

\begin{abstract}
Large hydrogen-isotopic (D/H) fractionations between lipids and growth water have been observed in most organisms studied to date. These fractionations are generally attributed to isotope effects in the biosynthesis of lipids, and are frequently assumed to be approximately constant for the purpose of reconstructing climatic variables. Here, we report $D / H$ fractionations between lipids and water in 4 cultured members of the phylum Proteobacteria, and show that they can vary by up to $500 \%$ in a single organism. The variation cannot be attributed to lipid biosynthesis as there is no significant change in these pathways between cultures, nor can it be attributed to changing substrate $\mathrm{D} / \mathrm{H}$ ratios. More importantly, lipid/water D/H fractionations vary systematically with metabolism: chemoautotrophic growth (approximately -200 to $-400 \%$ ), photoautotrophic growth $(-150$ to $-250 \%$ ), heterotrophic growth on sugars ( 0 to $-150 \%$ ), and heterotrophic growth on TCA-cycle precursors and intermediates $(-50$ to $+200 \%)$ all yield different fractionations. We hypothesize that the $\mathrm{D} / \mathrm{H}$ ratios of lipids are controlled largely by those of NADPH used for biosynthesis, rather than by isotope effects within the lipid biosynthetic pathway itself. Our results suggest that different central metabolic pathways yield NADPH-and indirectly lipids-with characteristic isotopic compositions. If so, lipid $\delta \mathrm{D}$ values could become an important biogeochemical tool for linking lipids to energy metabolism, and would yield information that is highly complementary to that provided by ${ }^{13} \mathrm{C}$ about pathways of carbon fixation.
\end{abstract}

fatty acids | fractionation | metabolism | hydrogen isotopes

$\mathbf{T}^{\mathrm{T}}$ he hydrogen-isotopic composition $\left({ }^{2} \mathrm{H} /{ }^{1} \mathrm{H}\right.$ or $\mathrm{D} / \mathrm{H}$ ratio, commonly expressed as a $\delta \mathrm{D}$ value) of lipids is being explored by scientists with diverse interests, including the origins of natural products $(1,2)$, biogeochemical cycles $(3)$, petroleum systems (4), and paleoclimate (5-7). Because the $\mathrm{D} / \mathrm{H}$ ratios of lipids are generally conserved over $\approx 10^{6}$-year time scales $(8)$, they are a potentially useful tracer of biogeochemical pathways and processes in the environment. Most research to date has focused on higher plants, in which environmental water is the sole source of external hydrogen and consequently provides primary control over the $\mathrm{D} / \mathrm{H}$ ratio of biosynthesized lipids (9). Although $\delta \mathrm{D}$ values for plant lipids and environmental water are generally well correlated, they are also substantially offset from each other. The biochemical basis for this lipid/water fractionation is not well understood. It is generally assumed to arise from a combination of isotope effects during photosynthesis and the biosynthesis of lipids (9-12), and is often treated as approximately constant to reconstruct isotopic compositions of environmental water as a paleoclimate proxy.

There is, however, mounting evidence that the net $\mathrm{D} / \mathrm{H}$ fractionation between lipids and water can vary by up to $150 \%$ in plants, even in the same organism (12-16). Modest fractionations associated with fatty acid elongation and desaturation have been documented $(2,14,15)$ but are unlikely to account for all of the observed variability. Recent surveys of lipids in marine environments have hinted at even greater variability in isotopic compositions. Jones et al. (17) measured fatty acids extracted from coastal marine particulate organic matter (POM) and found $\delta \mathrm{D}$ values ranging from -73 to $-237 \%$. Measurements of lipids from marine sediments have extended this range from -32 to $-348 \%$ o for lipids with $n$-alkyl skeletons and -148 to $-469 \%$ for those with isoprenoid skeletons (18). Because the lipids measured by both studies likely derive from marine organisms inhabiting seawater of essentially constant $\delta \mathrm{D}$ value $(\approx 0 \%)$, such differences cannot be due to varying environmental water. Rather, they must relate to more fundamental differences in metabolism.

Culture studies, although limited in number, support the occurrence of highly variable $\mathrm{D} / \mathrm{H}$ fractionations. Hydrocarbons produced by the green alga Botryococcus braunii were depleted in D relative to growth water by 197 to $358 \%$ (16). Fatty acids from the aerobic methanotroph Methylococcus capsulatus (19) were depleted by 20 to $70 \%$, whereas those in the sulfatereducing chemoautotroph Desulfobacterium autotrophicum were depleted by 190 to $360 \%$ (20). The most strongly fractionating organism reported to date is an $\mathrm{H}_{2}+\mathrm{CO}_{2}$ using acetogen, Sporomusa sp. DSM 58, which produced fatty acids with depletions in D of nearly $400 \%$ (21).

Some of this reported variability can be ascribed to systematic differences between lipids with $n$-alkyl versus isoprenoid skeletons. Isoprenoid lipids are typically D depleted relative to $n$-alkyl lipids by $100 \%$ or more, a pattern now widely confirmed in both culture $(13,16,19)$ and environmental samples $(12,18)$. However, significant variability within single classes of lipids (e.g., fatty acids) cannot be explained because the chemical mechanisms of lipid biosynthesis are strongly conserved across most bacterial and eukaryotic phyla (22-25). Thus, many important questions linger. Do $\mathrm{D} / \mathrm{H}$ fractionations associated with different metabolic lifestyles (i.e., photoautotrophy, chemoautotrophy, or heterotrophy) systematically differ? Do they differ in bacteria versus eukaryotes? Perhaps most fundamentally, how and why do biosynthetic processes fractionate hydrogen isotopes at the molecular level and lead to lipids with such diverse isotopic compositions? These questions lie at the heart of our ability to use and interpret lipid $\delta \mathrm{D}$ values from all types of environmental samples. To explore such issues, we measured lipid $\mathrm{D} / \mathrm{H}$ fractionations in 4 metabolically versatile bacteria grown under photoautotrophic, photoheterotrophic, chemoautotrophic, and heterotrophic conditions on a range of carbon sources metabolized by different pathways of central metabolism.

\footnotetext{
Author contributions: X.Z. and A.L.S. designed research; X.Z., A.L.G., and A.L.S. performed research; X.Z. analyzed data; and X.Z. and A.L.S. wrote the paper.

The authors declare no conflict of interest.

This article is a PNAS Direct Submission.

See Commentary on page 12565.

${ }^{1}$ To whom correspondence should be addressed. E-mail: als@gps.caltech.edu.
}

This article contains supporting information online at www.pnas.org/cgi/content/full/ 0903030106/DCSupplemental. 
Table 1. Summary of culture experiments

\begin{tabular}{|c|c|c|c|c|}
\hline Organism-substrate & $\delta D_{s}, * \% o$ & $\delta \mathrm{D}_{w_{\imath}}{ }^{\dagger} \%$ & Growth rate, ${ }^{\ddagger} \mathrm{h}^{-1}$ & Cultures ${ }^{\S}$ \\
\hline \multicolumn{5}{|l|}{ C. oxalaticus } \\
\hline oxalate & - & -68.6 to +218.3 & 0.29 & Co1-I,II,III,IV \\
\hline oxalate & - & -68.6 to +218.3 & 0.28 & Co2-I,II,III,IV \\
\hline formate & 972 & -68.6 to +218.3 & 0.33 & Co3-I,II,III,IV \\
\hline acetate & -76 & -68.6 to +218.3 & 0.50 & Co4-I,II,III,IV \\
\hline succinate & -97 & -64.3 to +214.1 & 0.60 & Co5-I,II,III,IV \\
\hline succinate & -97 & +41.1 to +214.1 & NA & Co6-II,III,IV \\
\hline \multicolumn{5}{|l|}{ C. necator } \\
\hline formate & 972 & -68.3 & 0.17 & Cn1-I \\
\hline fructose & -22 & -65.5 & 0.34 & $\mathrm{Cn} 2-\mathrm{I}$ \\
\hline gluconate & NA & -68.1 & 0.36 & Cn3-I \\
\hline pyruvate & -12 & -64.4 & 0.61 & Cn4-I \\
\hline acetate & -76 & -68.5 & 0.35 & Cn5-I \\
\hline succinate & -97 & -68.6 & 0.48 & Cn6-I \\
\hline succinate & -97 & -68.6 & NA & Cn7-I \\
\hline \multicolumn{5}{|l|}{ E. coli } \\
\hline glucose & -60 & -61.9 & 0.64 & Ec1-I \\
\hline gluconate & NA & -62.2 & 0.57 & Ec2-I \\
\hline pyruvate & -12 & -68.1 & 0.37 & Ec3-I \\
\hline acetate & -76 & -62.4 & 0.31 & Ec4-I \\
\hline glucose & -60 & -60.0 to +314.0 & 0.66 & Ec5-I,II,III,IV \\
\hline LB & NA & -60.0 to +152.0 & NA & Ec6-I,II,III \\
\hline \multicolumn{5}{|l|}{ R. palustris } \\
\hline acetate & NA & -53.6 & 0.10 & Rp1-I \\
\hline acetate, light & NA & -53.6 & 0.068 & Rp2-I \\
\hline $\mathrm{CO}_{2}$, light & - & -53.6 & 0.015 & Rp3-I \\
\hline
\end{tabular}

${ }^{*} \delta \mathrm{D}$ of non-exchangeable C-bound $\mathrm{H}$ in the growth substrate (see SI Methods for calculation details). Uncertainties are likely $<20 \%$. - indicates a substrate with no $H$, NA, not available.

${ }^{\dagger} \delta \mathrm{D}$ of culture medium before inoculation. Average analytical uncertainty $(1 \sigma)$ is $0.7 \%$. Range refers to the span of values covered by 4 replicate cultures, each differing by $\leq 100 \%$.

${ }^{\ddagger} 1 \sigma$ was $\leq 0.04$ for $n \geq 2$ cultures in experiments Co1-Co5.

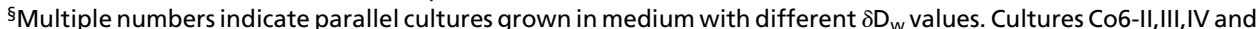
$\mathrm{Cn7-I} \mathrm{were} \mathrm{harvested} \mathrm{in} \mathrm{stationary} \mathrm{phase,} \mathrm{all} \mathrm{others} \mathrm{were} \mathrm{harvested} \mathrm{in} \mathrm{exponential} \mathrm{phase.} \mathrm{OD} \mathrm{values} \mathrm{at} \mathrm{harvest} \mathrm{are}$ in Fig. S1.

\section{Results}

Cultures and Fatty Acids. Four species of bacteria, chosen to provide a sampling of metabolic diversity, were grown in batch culture on varying substrates (Table 1, see SI Methods for details). Cupriavidus oxalaticus str. OX1 and C. necator str. H16 are facultative chemoautotrophic $\beta$-Proteobacteria commonly found in soil and freshwater environments (26), and were grown as aerobic heterotrophs and chemoautotrophs. The model organism Escherichia coli K-12 str. MG1655, an obligate heterotrophic $\gamma$-Proteobacterium, was grown aerobically. The purple non-sulfur anoxygenic phototroph Rhodopseudomonas palustris str. TIE- 1 is an $\alpha$-Proteobacterium and was grown under anaerobic photoautotrophic, anaerobic photoheterotrophic, and aerobic heterotrophic conditions. Organic substrates were chosen based on their catabolic relationship to the different pathways of central metabolism. They include those that feed into glycolysis (glucose, fructose, gluconate, pyruvate), the tricarboxylic acid (TCA) cycle (acetate, succinate), and chemoautotrophic* metabolism [formate, oxalate (27-29)].

Most cultures were harvested during exponential growth (Fig. S1). Fatty acids were solvent-extracted, derivatized as methyl esters, and quantified by gas chromatography/mass spectrometry

*Growth on oxalate is classically regarded as heterotrophic, not chemoautotrophic, metabolism. However, conservation of energy during growth is similar to that on formate in that 1-carbon reactions form the basis for generation of reducing power, analogous to "true" chemoautotrophy (e.g., growth on $\left.\mathrm{H}_{2}+\mathrm{CO}_{2}\right)(29,30)$. Hence we refer here to growth on formate and oxalate as chemoautotrophic for the purpose of describing hydrogen, rather than carbon, metabolism.
(GC/MS; Table S1). The most abundant fatty acids in $C$. oxalaticus and $C$. necator were palmitic (16:0), palmitoleic (16:1), and oleic (18:1) acids. An additional fatty acid, cyclopropylheptadecanoic acid (cyc-17), was abundant in E. coli. R. palustris produced significant amounts of 18:1, 18:0 (stearic acid), and 16:0 fatty acids. Relative abundances of fatty acids varied by $<35 \%$ between cultures of each bacterial species, with no systematic relationship between growth substrate and fatty acid abundance (Table S1).

Values of $\delta \mathrm{D}$ for individual fatty acids varied widely between cultures $(-362$ to $+331 \%$ ), but typically by less than $\approx 30 \%$ o between different fatty acids from the same culture (Table S2). For simplicity we report and discuss the $\delta \mathrm{D}$ values for palmitic acid as representative of each culture, both because it was present in every organism and because it was generally the most abundant fatty acid.

Growth on Different Substrates Leads to Varying Fractionation Between Lipids and Water. Fig. 1 summarizes current and previous culture data and shows that the net $\mathrm{D} / \mathrm{H}$ fractionations between lipids and culture water vary in all analyzed strains. The 2 Cupriavidus strains, which have the most metabolically versatile carbon metabolisms, exhibited the largest variability (up to $500 \%$ ). This is the largest range of isotopic fractionations yet recorded for any individual organism, and includes instances of both $\mathrm{D}$ enrichment and $\mathrm{D}$ depletion relative to water. The observed range of fractionations is substantially larger than has been previously observed in environmental samples, and-if expressed in nature-would have the potential to explain all such 


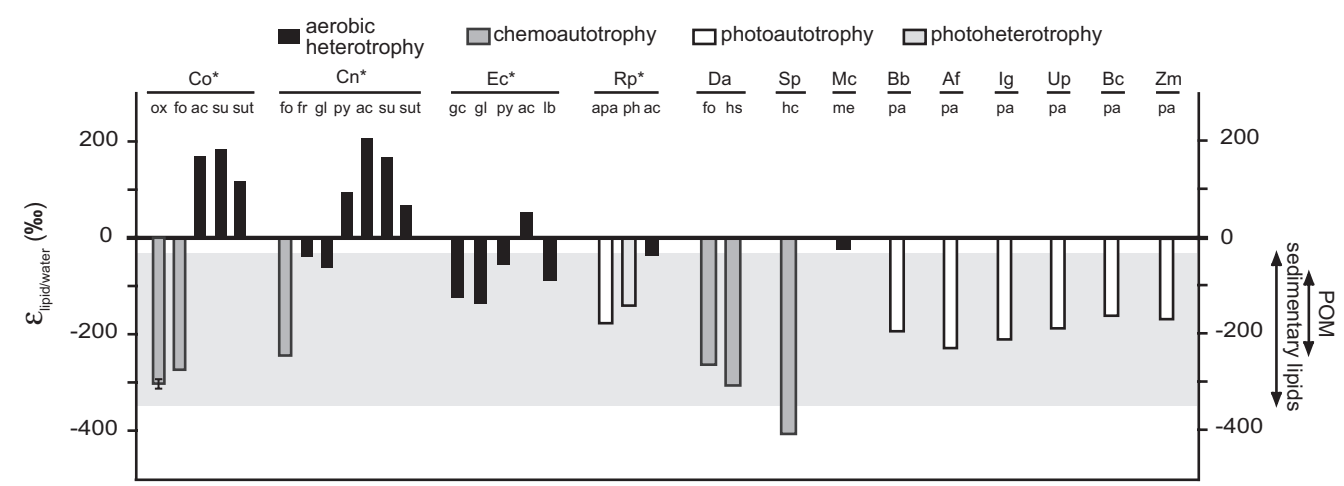

Fig. 1. Summary of $\mathrm{D} / \mathrm{H}$ fractionations between fatty acids and water observed in culture experiments, native specimens, and marine organic matter fractions. Plotted fractionations are based on the average $\delta \mathrm{D}$ value of palmitic acid, or the fatty acid/alkane of nearest chain length, in the culture with water $\delta \mathrm{D}$ closest to $0 \%$. Bacterial cultures from this study $\left(^{*}\right)$ are C. oxalaticus (Co), C. necator (Cn), E. coli (EC), and R. palustris (Rp). Organisms from other studies (13, 16, 19-21, 61) are cultured bacteria $D$. autotrophicum (Da), Sporumusa sp. (Sp), and M. capsulatus (Mc), cultured phytoplankton B. braunii (Bb), Alexandrium fundyense (Af), Isochrysis galbana (Ig), and natural specimens of brown alga Undaria pinnatifida (Up), red alga Binghamia californica (BC), and seagrass Zostera marina (Zm). The gray box covers the range of fractionations observed in marine POM and sediments $(17,18)$. Growth substrates are oxalate (ox), formate (fo), fructose (fr), glucose (gc), gluconate (gl), pyruvate (py), acetate (ac), succinate (su, sut), LB (lb), $\mathrm{H}_{2}+\mathrm{CO}_{2}(\mathrm{hc}), \mathrm{H}_{2}+\mathrm{SO}_{4}{ }^{2-}(\mathrm{hs})$, methane (me), acetate+light (ph), $\mathrm{CO}{ }_{2}+\mathrm{light}$ (pa), $\mathrm{S}_{2} \mathrm{O}_{3}{ }^{2-}+\mathrm{CO}_{2}+$ light (apa). Error bars for culture $\mathrm{Co}^{*}$-ox are the standard deviation $( \pm 1 \sigma)$ for 4 sets of biological replicates; other cultures were not replicated. Typical analytical uncertainties are $\pm 3.5 \%$ for all cultures.

environmental $\mathrm{D} / \mathrm{H}$ variability. At the same time, lipid/water fractionation during anoxygenic photoautotrophic growth of $R$. palustris was within the range commonly observed for plants. For heterotrophic cultures, the $\delta \mathrm{D}$ values of most supplied organic substrates $\left(\delta \mathrm{D}_{\mathrm{s}}\right)$ differ by $<100 \%$ (Table 1$)$ and cannot explain the range of observed lipid $\delta \mathrm{D}$ values. For example, growth of $E$. coli on glucose $\left(\delta \mathrm{D}_{\mathrm{s}}=-60 \%\right)$ led to $\mathrm{D}$ depletion of lipids relative to both water and substrate, whereas growth on acetate $\left(\delta \mathrm{D}_{\mathrm{s}}=-76 \%\right.$ ) led to $\mathrm{D}$ enrichment.

Our data show a strong correspondence between the pathways of substrate metabolism and lipid $\delta \mathrm{D}$ values. Growth on formate or oxalate, catabolized through 1-carbon reactions $(29,30)$, yielded lipids depleted in D by 200 to $300 \%$ relative to water (Fig. 1, Table S2). Heterotrophic growth on sugars and photoautotrophic growth on $\mathrm{CO}_{2}$ produced lipids depleted by 50 to $190 \%$ relative to water. Growth on a direct precursor (acetate) and intermediate (succinate) of the TCA cycle yielded lipids that were generally D-enriched relative to water $(-50$ to $+200 \%)$, with growth phase modulating the level of enrichment (compare "su" vs. "sut" in Fig. 1). These patterns are most strongly exhibited in the 2 Cupriavidus strains, but are also present in $E$. coli and $R$. palustris.

Manipulation of Growth Water $\delta D$ : Fractionation Factor Curves. Additional information on the biochemical causes of these variable fractionations can be deduced from experiments in which the isotopic composition of culture water is experimentally manipulated (19). Conceptually, the net fractionations between lipids and each external $\mathrm{H}$ source (i.e., water and organic substrate) can be treated as distinct, yielding the isotopic mass balance

$$
R_{1}=X_{\mathrm{w}} \alpha_{1 / \mathrm{w}} R_{\mathrm{w}}+\left(1-X_{\mathrm{w}}\right) \alpha_{1 / \mathrm{s}} R_{\mathrm{s}},
$$

where $R_{\mathrm{l}}, R_{\mathrm{w}}$, and $R_{\mathrm{s}}$ denote the $\mathrm{D} / \mathrm{H}$ ratios of lipids, water, and substrates, respectively (31). $X_{\mathrm{w}}$ is the mole fraction of lipid H derived from external water, whereas $\alpha_{1 / \mathrm{w}}$ and $\alpha_{1 / \mathrm{s}}$ represent the net isotopic fractionations associated with uptake and utilization of water and substrate hydrogen, respectively. Eq. 1 represents the overall isotopic relationship between lipids and external sources of $\mathrm{H}$, and does not imply that the sources must be directly involved in lipid biosynthesis (e.g., glucose may contribute $\mathrm{H}$ to lipids by way of metabolic intermediates even though it does not directly participate in the biosynthetic reactions). Measurements of $R_{\mathrm{w}}, R_{\mathrm{s}}$, and $R_{1}$ for parallel cultures in which only 1 parameter,
$R_{\mathrm{w}}$, is varied experimentally form the basis for regression of $R_{1}$ on $R_{\mathrm{w}}$. This yields a unique slope and intercept that can be used to constrain the relevant fractionations.

To this end, $C$. oxalaticus and E. coli were grown on glucose, acetate, succinate, formate, oxalate, and Lysogeny broth (LB) using waters with $\delta \mathrm{D}$ values ranging from -68 to $+314 \%$. Strong linear relationships $\left(R^{2} \geq 0.98\right)$ between $\delta \mathrm{D}$ values of fatty acids and water were obtained for all such experiments (Fig. 2), implying that the uncertainty in $\delta \mathrm{D}$ values for each culture is minimal (probably $<20 \%$ ). This is consistent with several "true" biologic replicates grown in identical waters, for which $\delta \mathrm{D}$ values differed by $<11 \%$ (1\%).

For cultures where $X_{\mathrm{w}}$ is known, $\alpha_{1 / \mathrm{w}}$ and $\alpha_{1 / \mathrm{s}}$ can be calculated directly from the slope and intercept of the regression. Oxalate carries no $\mathrm{H}$ at physiological $\mathrm{pH}$, so $X_{\mathrm{w}}=1$ for $C$. oxalaticus

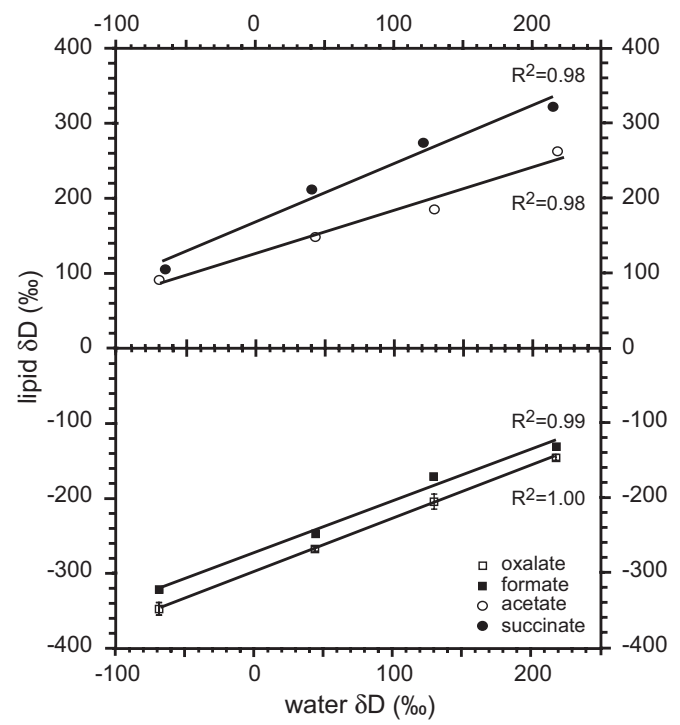

Fig. 2. Regressions of $\delta \mathrm{D}$ values for palmitic acid versus water for $C$. oxalaticus grown on oxalate, formate, acetate, and succinate. Error bars represent $1 \sigma$ uncertainty for biological replicates. Data for other lipids are in Fig. S2 and Table S3. Each regression provides constraints on fractionations that can be described most succinctly as a single fractionation curve (see Fig. 3). 


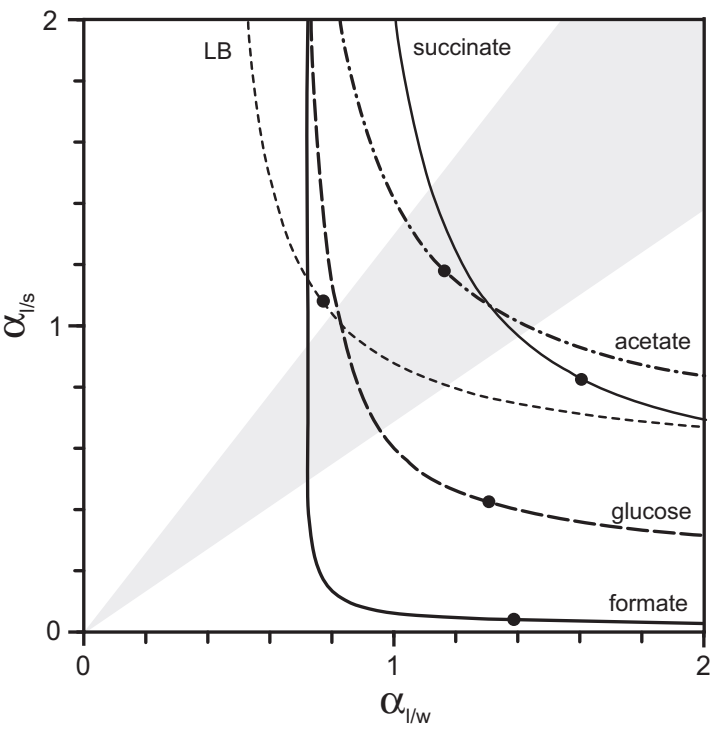

Fig. 3. Fractionation factor curves for palmitic acid in C. oxalaticus grown on formate, acetate, and succinate, and in E. coli grown on glucose and LB. Each curve represents the set of all possible combinations of $\alpha_{\mid / /}$and $\alpha_{\mid / w}$ satisfying the constraints imposed by parallel cultures with differing $R_{w}$ (i.e., 1 linear regression in Fig. 2). Filled circles indicate values corresponding to $X_{\mathrm{w}}=0.5$ Gray shaded area defines up to $600 \%$ variation between $\alpha_{l / 5}$ and $\alpha_{I / w}$. Increases in the true value of $\alpha_{\mathrm{l} / \mathrm{s},}, \alpha_{\mathrm{l} / \mathrm{w}}$, and $X_{\mathrm{w}}$ shift curves up, to the right, or down a diagonal as detailed in Fig. S3.

growing on oxalate. The corresponding value of $\alpha_{1 / \mathrm{w}}$ ranged from 0.64 to 0.73 for different fatty acids (Table S3). Growth on formate yielded nearly identical results, even though formate is a potential source of $\mathrm{H}$. We infer that $\mathrm{H}$ on formate exchanges with water to preclude the transmission of substrate $\mathrm{H}$ to fatty acids, in accord with our understanding of formate metabolism (29) and the recent results of Campbell et al. (20).

If $X_{\mathrm{w}}$ is unknown, as is the case for most heterotrophic growth conditions, then a unique solution for $X_{\mathrm{w}}, \alpha_{\mathrm{l} / \mathrm{w}}$, and $\alpha_{\mathrm{l} / \mathrm{s}}$ is not possible (31). However, the results of each regression can be depicted as a curve relating $\alpha_{1 / \mathrm{s}}$ to $\alpha_{1 / \mathrm{w}}$, with each point on the curve representing a different possible combination of values for $X_{\mathrm{w}}, \alpha_{1 / \mathrm{w}}$, and $\alpha_{1 / \mathrm{s}}$ (Fig. 3). These relationships, which we term "fractionation curves," are unique for each set of culture conditions and provide a useful means for comparison even while the true values of $X_{\mathrm{w}}, \alpha_{1 / \mathrm{w}}$, and $\alpha_{\mathrm{l} / \mathrm{s}}$ remain unknown. A basic understanding of the behavior of such curves aids in their interpretation: When comparing 2 fractionation curves, representing 2 different culture conditions, an increase in the true value of $\alpha_{1 / \mathrm{w}}$ between conditions will shift the curves horizontally to the right; an increase in the true value of $\alpha_{1 / \mathrm{s}}$ will shift them vertically upward; and an increase in $X_{\mathrm{w}}$ will shift them diagonally downward and to the right (see Fig. S3).

Two key inferences can be drawn from the fractionation curves in Fig. 3. First, within a plausible range of values for the 2 fractionation factors ( 0.5 to 2.0$)$, equivalent to $\pm 1,000 \%$ and 2-fold larger than any net fractionations yet measured for biosynthetic processes, many fractionation curves do not intersect. This is possible only if both $\alpha_{1 / \mathrm{w}}$ and $\alpha_{1 / \mathrm{s}}$ vary between conditions (e.g., compare C. oxalaticus on formate vs. E. coli on glucose vs. $C$. oxalaticus on succinate). Changes in fractionation associated with the assimilation of substrate, $\alpha_{1 / \mathrm{s}}$, cannot by itself explain our results, and it is clear that the concept of a nearly constant lipid/water fractionation must be discarded. Because $\alpha_{1 / \mathrm{w}}$ changes even in a single organism, whereas pathways of lipid biosynthesis are not known to change significantly with growth on different substrates, we infer that the magnitude of $\alpha_{1 / \mathrm{w}}$ is not set primarily by lipid biosynthetic reactions.

Second, fractionation curves for $E$. coli growth on glucose and LB are offset along a diagonal, and thus are likely related by similar fractionations ( $\alpha$ values) with a decrease in $X_{\mathrm{w}}$ from glucose to LB. This is consistent with the assimilation of preformed cell constituents by cells grown on the complex LB medium, leading to smaller $X_{\mathrm{w}}$. Curves for growth on acetate and succinate also appear related by similar fractionations with a decrease in $X_{\mathrm{w}}$ from succinate to acetate, and are consistent with the role of acetate as a direct precursor for lipid biosynthesis.

Similarity of Lipid-Substrate and Lipid-Water Fractionations. Although it is convenient to treat the net lipid/water and lipid/ substrate fractionations as independent, this distinction is probably artificial. Approximately $3 / 4$ of fatty acid $\mathrm{H}$ derives from central metabolites (acetate or NADPH; see Discussion). H derived from water and growth substrates is comingled in these and virtually all other metabolites because many common classes of reactions (isomerization, hydrolysis, rearrangement, exchange) lead to significant scrambling of C-bound $\mathrm{H}$ (32). At this point the 2 "external" sources of $\mathrm{H}$ should be affected by many of the same reactions and thus the same isotope effects. The possibility of markedly different values for $\alpha_{1 / \mathrm{s}}$ and $\alpha_{1 / \mathrm{w}}$ is therefore difficult to envision. Indeed, having widely different fractionations for water and substrate $\mathrm{H}$ would practically require that these two $\mathrm{H}$ pools remain metabolically distinct, but they do not. Although a strong covariance of $\alpha_{1 / \mathrm{w}}$ and $\alpha_{1 / \mathrm{s}}$ does reduce the utility of our isotopic labeling approach, there is also a substantial benefit in that the inverse problem-inferring biochemical processes from measured lipid $\delta \mathrm{D}$ values-is made much easier. The strong correspondence of metabolic pathways and lipid $\delta \mathrm{D}$ values implied by Fig. 1 would be highly improbable if $\alpha_{1 / \mathrm{s}}$ and $\alpha_{1 / \mathrm{w}}$ both varied widely and independently.

To extract further insight from Fig. 3, we therefore assume that $\alpha_{1 / \mathrm{s}}$ and $\alpha_{1 / \mathrm{w}}$ for any single culture differ from each other by $\leq 600 \%$ (i.e., within the gray region in Fig. 3). This is an arbitrary limit, chosen to encompass the $\approx 500 \%$ range of fractionations we observe, but more conservative limits would yield similar conclusions. Probable limits for $X_{\mathrm{w}}, \alpha_{\mathrm{l} / \mathrm{w}}$, and $\alpha_{\mathrm{l} / \mathrm{s}}$ can then be calculated: formate $(>95 \%, 0.73, \mathrm{NA})$, glucose (68-80\%, 0.80-0.95, 0.67-1.05), LB (33-47\%, 0.80-1.15, 0.82$1.05)$, succinate $(58-70 \%, 1.13-1.38,0.98-1.48)$, and acetate $(40-56 \%, 1.03-1.43,1.0-1.35)$. The fact that both $\alpha_{1 / \mathrm{w}}$ and $\alpha_{1 / \mathrm{s}}$ vary from $\mathrm{D}$ depletion $(\alpha<1)$ to $\mathrm{D}$ enrichment $(\alpha>1)$ strongly supports our contention that variability in fatty acid $\delta \mathrm{D}$ values cannot be explained solely by modulation of a single fractionating step in their biosynthesis.

Four conclusions arise from this analysis. First, more $\mathrm{H}$ is transmitted from water to fatty acids when growing on sugars than on acetate or succinate. This may reflect greater exchange of $\mathrm{H}$ associated with sugar isomerization reactions, and/or the fact that acetate feeds directly into fatty acid biosynthesis. Second, no $\mathrm{H}$ is transmitted from "chemoautotrophic" substrates to lipids, including formate, oxalate, and $\mathrm{H}_{2}$. Third, the fractionations associated with different metabolic pathways are distinct but partially overlapping, in the order chemoautotrophy $<$ photoautotrophy $<$ heterotrophic growth on sugars $<$ growth on TCA-cycle substrates. This pattern of changing fractionations mirrors the observed shifts in fatty acid $\delta D$ values, and forms the basis for a plausible mechanistic link between lipid $\delta \mathrm{D}$ and metabolism. Fourth, the net lipid/water fractionation for a fatty acid is characteristic of a particular metabolism despite a wide possible range of $\delta \mathrm{D}$ values for potential organic substrates, because relatively little substrate $\mathrm{H}$ is incorporated into fatty acids. This means useful metabolic information can be extracted from the $\delta \mathrm{D}$ values of environmental lipids without the necessity of knowing or measuring $\delta \mathrm{D}$ values of precursor substrates. 


\section{Discussion}

Our results demonstrate that 4 metabolically diverse Proteobacteria produce fatty acids with $\delta \mathrm{D}$ values that vary systematically with the utilization of key metabolic pathways. The pattern is supported by previous culture studies of bacteria (19-21) and plants $(11,33)$ and is consistent with field observations. For example, fatty acids with odd carbon-numbered chains are substantially D-enriched (by up to $100 \%$ ) relative to those with even-numbered chains in marine POM (17). The former are attributed to heterotrophic bacteria, whereas the latter are likely the products of photosynthetic algae. Similar D enrichments of many bacterial fatty acids and all hopanols relative to their algal counterparts (i.e., even-numbered fatty acids and sterols) have also been observed in marine sediments (18). A 16:1 fatty acid with a $\delta \mathrm{D}$ value as low as $-348 \%$ was detected in those sediments and is apparently generated in situ within the zone of maximal sulfate reduction. Its occurrence and isotopic composition are consistent with origins from chemoautotrophic sulfatereducing bacteria (18). Studies of higher plants have shown several cases of D enrichment resulting from increased reliance on stored carbohydrates for growth and maintenance, and are consistent with a shift from photosynthesis to glycolysis as the primary metabolism $(32,34)$.

These similarities lead us to propose that the systematic variations reported here are a general feature resulting from the commonality of central metabolic pathways present in most microbes. Most of the same pathways are also present in higher life forms, although the resulting isotopic patterns will likely be complicated by compartmentalization and transport of lipids, metabolites, and water. Because we have sampled only a minute fraction of extant biota, further culture-based evidence is required to address these issues. In the mean time, confidence that we have observed a general phenomenon can be improved by understanding the mechanistic link between metabolism and $\mathrm{D} / \mathrm{H}$ fractionation in lipids. Although we do not yet have sufficient data to prove such a link, we suggest that fractionations accompanying the reduction of $\mathrm{NADP}^{+}$provide a plausibleand perhaps unavoidable-mechanism. The basis for this hypothesis is summarized next.

Sources of D/H Variability in Fatty Acids. Isotopic labeling studies of fatty acid biosynthesis in vitro provide a rough accounting of the $\mathrm{H}$ sources for fatty acids $(1,35,36)$. They indicate that the most important cellular $\mathrm{H}$ source is $\mathrm{NAD}(\mathrm{P}) \mathrm{H}$, providing $\approx 50 \%$ of fatty acid $\mathrm{H}$ (Fig. S4). In most cases this comes solely from NADPH, but in some organisms such as $E$. coli it derives equally from both NADPH and NADH $(22-24,37)$. The methyl group of acetyl-CoA $(25 \%)$ and water $(25 \%)$ are of lesser importance. Four possible sources of isotopic variability in fatty acids can then be considered: (i) fractionations associated with substrate uptake and utilization; (ii) the isotopic composition of cellular water, acetate, and NADPH used for biosynthesis; (iii) variations in fractionation associated with reactions of the fatty acid biosynthetic pathway itself, including transfer of $\mathrm{H}$ from water and/or NADPH to fatty acids; and (iv) fractionations downstream from fatty acid biosynthesis such as desaturation and cellular transport.

Analysis of fractionation curves (see Results) removes $i$ as a possibility. The lack of significant changes in fatty acid abundance and structure between culture conditions with very different lipid $\delta \mathrm{D}$ values rules out $i v$. Option $i i i$ is worth considering in some detail because multiple enzyme variants exist for 1 of the 2 reductive steps in fatty acid biosynthesis. $\beta$-ketoacyl ACP reductase transfers $\mathrm{H}$ from NADPH to odd-numbered carbon positions on the nascent fatty acid (step 4 in Fig. S4), whereas enoyl ACP reductase transfers $\mathrm{H}$ from either NADPH or NADH to even and odd positions (step 6 in Fig. S4) $(22,24)$. Only one type of $\beta$-ketoacyl ACP reductase (FabG) is known, but multiple variants (FabI, FabK, FabL) of enoyl reductase exist, sometimes in the same bacterium (23-25). Thus, differences in fractionations between flavin-free pyridine nucleotide-dependent enzymes like FabG and FabI versus flavoproteins like FabK might provide a mechanism for lipid $\mathrm{D} / \mathrm{H}$ variability. The former catalyze direct hydride $\left(\mathrm{H}^{-}\right)$transfer from $\mathrm{NAD}(\mathrm{P}) \mathrm{H}$ to fatty acids $(24,38$, 39 ), whereas in the latter $\mathrm{H}^{-}$is transferred via the flavin ring, which is susceptible to isotopic exchange with water $(36,40,41)$. Differing fractionations between the enzyme types are therefore plausible. However, E. coli has only 1 of each reductase enzyme (i.e., a single FabG and FabI) yet lipid/water fractionations still vary substantially $(23,37,42,43)$. C. necator has several putative $\beta$-ketoacyl ACP reductases and FabI-type enoyl ACP reductases (26), but fractionations in $C$. necator are similar to those of $E$. coli. Thus, current data are inconsistent with fatty acid biosynthetic enzymes causing large variability in fatty acid $\delta \mathrm{D}$ values.

This leads us to consider option $i$, the $\mathrm{H}$-isotopic composition of water, acetate, and $\mathrm{NAD}(\mathrm{P}) \mathrm{H}$, as sources for variability in fatty acid $\delta \mathrm{D}$ values. Variations in the $\mathrm{D} / \mathrm{H}$ ratio of intracellular water have been observed in rapidly-growing $E$. coli and were attributed to the accumulation of water derived from the oxidation of organic substrates (44). Given that substrate $\delta \mathrm{D}$ values did not approach $+200 \%$ or $-300 \%$ in our study, and no systematic relationship between growth rate and fatty acid $\delta \mathrm{D}$ value was observed, the influence of varying intracellular water $\delta \mathrm{D}$ can be ruled out. We eliminate acetate as a source for isotopic variability on the following grounds. When $C$. oxalaticus grows on oxalate, it synthesizes acetate by converting oxalate to 3-phosphoglycerate (Box 3 in Fig. S5) (29). In contrast, 3-phosphoglycerate is generated by $\mathrm{CO}_{2}$ fixation in the Calvin cycle when $C$. oxalaticus grows on formate (Box 7 in Fig. S5) (29). Thus, 2 very different modes of acetate synthesis (growth on oxalate vs. formate) yield similar fractionations, whereas synthesis of acetate by the same pathway of carbon fixation in different organisms (e.g., C. oxalaticus grown on formate and all higher plants) yields very different fractionations.

By process of elimination then, we arrive at the inference that the isotopic composition of $\mathrm{NAD}(\mathrm{P}) \mathrm{H}$ is likely responsible for observed variations in lipid/water fractionation. This conclusion is consistent both with the role of $\mathrm{NAD}(\mathrm{P}) \mathrm{H}$ as the major source of $\mathrm{H}$ in fatty acids, and with the ability of flavin-free reductases to transmit isotopic signals from $\mathrm{NAD}(\mathrm{P}) \mathrm{H}$ to fatty acids via hydride transfer reactions $(38,39)$. But why should the $\mathrm{H}$ isotopic composition of NAD $(\mathrm{P}) \mathrm{H}$ vary so greatly?

Isotopic Composition of NADPH. Biosynthetic reactions commonly require NADPH rather than $\mathrm{NADH}(27,28,45)$, thus we consider here only the cellular sources of NADPH for simplicity. Similar arguments apply to sources of NADH. In heterotrophic metabolism, the main sources of NADPH are the oxidative reactions catalyzed by glucose-6-phosphate dehydrogenase and 6-phosphogluconate dehydrogenase in the pentose phosphate pathway, isocitrate dehydrogenase and malic enzyme in the TCA cycle, and the NADH-NADPH converting transhydrogenase (Fig. S5) $(28,45)$. These oxidation reactions typically involve direct $\mathrm{H}^{-}$transfer from substrate to $\mathrm{NADP}^{+}(38,39,46,47)$. Thus, the isotopic composition of each reduced NADPH will depend on that of the reaction substrate plus any isotope effects associated with the $\mathrm{H}^{-}$transfer, and the total pool of NADPH will reflect the relative contributions of different pathways of energy metabolism. In oxygenic photoautotrophs, $\mathrm{NADP}^{+}$is reduced via the oxidation of water by ferredoxin-NADP oxidoreductase (48), providing yet another source of NADPH.

Isotope fractionations associated with many NADPHgenerating reactions have been studied in vitro. Although their magnitudes vary greatly (up to 3,500\%o, see Fig. S5) (49-52), it is not possible to confidently predict in vivo fractionations, and thus the isotopic composition of generated NADPH, from these 
data. In part this is because kinetic isotope effects will not be fully expressed as isotopic fractionations in committed pathways where the reactant is completely consumed (53). Nor can data from cultures be used, because even organisms grown on a single substrate generate NADPH via multiple pathways (54). Nevertheless, it is reasonable to expect that the known variability in enzymatic isotope effects will manifest itself as varying $\delta \mathrm{D}$ values for NADPH generated by the respective pathways.

The enrichment of $\mathrm{D}$ in lipids from cultures grown on acetate and succinate is particularly interesting, because all of the relevant NADPH-generating reactions have normal isotope effects that should result in depletion of $\mathrm{D}$. We consider 2 possible explanations here. First, D enrichments may arise during $\mathrm{NADP}^{+}$reduction in the TCA cycle. Both malic enzyme and isocitrate dehydrogenase generate NADPH via $\mathrm{H}^{-}$transfer from an $\mathrm{OH}-\mathrm{C}-\mathrm{H}$ position ( $\mathrm{C}-2$ in isocitrate and malate). The expression of (normal) isotope effects in these reactions should be limited, because there is only one $\mathrm{H}$ available for abstraction. However, in both cases the substrate $\mathrm{OH}-\mathrm{C}-\mathrm{H}$ group is generated by the upstream removal of $\mathrm{H}$ from a corresponding methylene $(\mathrm{H}-\mathrm{C}-\mathrm{H})$ position in the precursor molecule (succinate and citrate in Fig. S5). These upstream reactions are catalyzed by succinate dehydrogenase and aconitase. Both have been shown to express significant normal isotope effects $(50,52$, $55)$, and are present in $C$. necator and $E$. coli $(26,43)$. For example, an average kinetic fractionation of $4,400 \%$ was measured for the removal of pro- $R \mathrm{H}$ from methylene groups in succinate by flavoprotein succinate dehydrogenase (55). The scale of this isotope effect is consistent with that expected for other flavoproteins, which have been proposed to break $\mathrm{C}-\mathrm{H}$ bonds by H-tunneling mechanisms (56). These reactions should leave the remaining $\mathrm{OH}-\mathrm{C}-\mathrm{H}$ position very strongly enriched in $\mathrm{D}$, a signal that can be transferred to NADPH. A second possible route to $\mathrm{D}$ enrichment of NADPH is through the action of transhydrogenases. These balance overproduction of NADPH due to high TCA cycle flux by converting it to NADH (54). Kinetic fractionations for transhydrogenases are typically large (800 to $3,500 \%$ ) (51), and should also leave the remaining NADPH strongly D-enriched.

The preceding discussion indicates that $(i) \mathrm{NAD}(\mathrm{P}) \mathrm{H}$ is the source for $\approx 50 \%$ of fatty acid hydrogen, (ii) the $\mathrm{D} / \mathrm{H}$ ratios of $\mathrm{NAD}(\mathrm{P}) \mathrm{H}$ generated in different metabolic pathways probably vary over a large range, and (iii) those isotopic signals can be transmitted to fatty acids via hydride transfer reactions. Given these constraints, we should ask whether a link between metabolism and fatty acid $\delta \mathrm{D}$ values can in any way be avoided? Practically the only possibilities are if the relative fluxes of different NADPH-generating pathways remain constant, or if isotopic exchange homogenizes the NADPH pool with water. The former can be dismissed because metabolic flux studies in E. coli conclusively show that NADPH sources vary significantly during growth on different substrates $(54,57,58)$.

The possibility of hydrogen exchange warrants further consideration because the relevant $\mathrm{H}$ position in $\mathrm{NAD}(\mathrm{P}) \mathrm{H}$ is moderately acidic. Experiments with D-labeled NADPH added to purified fatty acid biosynthetic enzymes in vitro showed complete conservation of the label in resultant fatty acids, whereas addition to crude cell extracts showed loss of the label (35). The latter result was attributed to isotope exchange via flavoproteins unrelated to lipid biosynthesis in the extract. If isotopic exchange of NADPH also occurs in vivo, it could serve to partially or entirely mute the fractionations accompanying NADPH production. As a concrete example, the slower growth rate of $R$. palustris might explain the smaller fractionations exhibited by this organism growing on acetate relative to $C$. oxalaticus. The extent to which this process is relevant in environmental samples is currently unknown, and must depend on turnover times for NADPH in growing cells. Although very little is known about the turnover of NADPH specifically, we note that the time scales for in vitro experiments $(\approx 5 \mathrm{~h})$ are quite long compared with typical turnover times for many common metabolic intermediates.

\section{Conclusions}

Existing culture and field data indicate that the $\mathrm{D} / \mathrm{H}$ ratios of lipids vary substantially with growth conditions, and are systematically related to pathways of central metabolism. Organisms growing on heterotrophic substrates exhibit lipid/water fractionations ranging between approximately -150 to $+200 \%$, photoautotrophic growth yields moderate D depletions $(-150$ to $-250 \%$ ), whereas chemoautotrophic growth yields very strong D depletions of -200 to $-400 \%$. We suggest that fractionations in the various pathways that reduce $\mathrm{NADP}^{+}$are the likely source of these variations. However, regardless of mechanism, such patterns hold enormous potential as biogeochemical tracers if they are shown to be widespread. This is particularly so given that the information provided by lipid $\delta \mathrm{D}$ values would be highly complementary to that encoded by molecular structure and $\mathrm{C}$ and $\mathrm{N}$ stable isotopes. Whereas ${ }^{13} \mathrm{C}$ largely records carbon fixation pathways in autotrophs, ${ }^{2} \mathrm{H}$ will respond to pathways of energy conservation. In heterotrophs, ${ }^{13} \mathrm{C}$ and ${ }^{15} \mathrm{~N}$ generally reflect the history of substrate transfers through successive trophic levels, whereas ${ }^{2} \mathrm{H}$ could provide a snapshot of the metabolic pathways used for energy generation by individual organisms. The ability to connect lipids with energy metabolism could find numerous applications, from assessing the in situ metabolic lifestyle of facultative heterotrophs, to identifying modern and ancient communities based on chemoautotrophy, to apportioning the relative contributions of primary production and heterotrophic recycling to sedimentary organic matter.

\section{Materials and Methods}

For details, see SI Methods.

Culture Strains and Growth. Cupriavidus oxalaticus str. OX1, Cupriavidus necator str. H16, Escherichia coli K-12 str. MG1655, and Rhodopseudomonas palustris str. TIE-1 were grown in batch culture on a variety of substrates (Table 1). The $\delta D$ of culture water was manipulated for $C$. oxalaticus and $E$. coli cultures by volumetrically diluting $99.9 \%$ purity $\mathrm{D}_{2} \mathrm{O}$ with distilled deionized water. Substrate $\delta D$ was not manipulated. Defined carbon sources were provided at $15 \mathrm{mM}$ (except for $22.2 \mathrm{mM}$ glucose in Ec5) for minimal media cultures of Cupriavidus and E. coli. Undefined carbon source LB was used for Ec6 cultures. $R$. palustris was cultivated in minimal medium with $20 \mathrm{mM}$ thiosulfate for photoautotrophy and $20 \mathrm{mM}$ acetate for both photoheterotrophy and aerobic heterotrophy. All media were $0.2-\mu \mathrm{m}$ filter sterilized and inoculated with single colonies from rich media plates. Culture purity was checked by microscopy, colony morphology on plates, and-for Cupriavidus - the ability to grow on oxalate. Optical density (OD) at $600 \mathrm{~nm}$ (Cary 50 Bio, UV-Vis Spec) was used in conjunction with growth curve data (Fig. S1) to harvest cultures at a specific growth phase, generally mid-log phase. To harvest, $\approx 0.4 \mathrm{~L}$ was centrifuged for 20 min at 4,500 $\times g$, yielding cell pellets ranging from 0.2 to $0.7 \mathrm{~g}$ of wet mass. These were stored at $-20^{\circ} \mathrm{C}$ before extraction.

Lipid Extraction and Quantification. Frozen cell pellets were lyophilized, then $\approx 20 \mathrm{mg}$ of biomass was simultaneously transesterified and extracted in hexane/ methanol/acetyl chloride at $100{ }^{\circ} \mathrm{C}$ for $10 \mathrm{~min}$ (59). The extract was concentrated under $\mathrm{N}_{2}$ at room temperature. Fatty acid methyl esters (FAMEs) were analyzed by gas chromatography/mass spectrometry (GC/MS) on a Thermo-Scientific Trace/ DSQ with a ZB-5ms column and PTV injector operated in splitless mode. Peaks were identified by comparison of mass spectra and retention times to authentic standards and library data. Relative abundances were calculated based on peak areas from the total ion chromatogram without further calibration. They are thus only semiquantitative, but still serve to demonstrate that fatty acid compositions did not change appreciably with growth substrate.

Isotopic Analyses. The $\delta \mathrm{D}$ values of the most abundant FAMEs were measured by GC/pyrolysis/isotope-ratio mass spectrometry (IRMS) on a Thermo-Scientific Delta ${ }^{+}$XP. Chromatographic conditions were identical as for GC/MS analyses, and peaks were identified by retention order and relative height. Data are reported in the conventional $\delta$ D notation versus the VSMOW standard, and are corrected 
for the addition of methyl $\mathrm{H}$ in the derivative. The root-mean-square (RMS) error of all external standards analyzed with these samples was $2.9 \%$. Typical precision $(1 \sigma)$ for replicate analyses of analytes was $3.4 \%$. The $\delta \mathrm{D}$ values of culture media, subsampled (1 $\mathrm{mL}$ ) before inoculation, were measured on a Los Gatos Research DLT-100 liquid water isotope analyzer. Samples were calibrated against 3 working standards with $\delta \mathrm{D}$ values ranging from -59 to $+290 \%$. These were in turn calibrated against the VSMOW, GISP, and SLAP international standards (60). Average precision was $0.7 \%(1 \sigma)$. $\delta \mathrm{D}$ values of nonexchangeable $\mathrm{H}$ in selected

1. Robins $\mathrm{RJ}$, et al. (2003) Measurement of ${ }^{2} \mathrm{H}$ distribution in natural products by quantitative ${ }^{2} \mathrm{H}$ NMR: An approach to understanding metabolism and enzyme mechanism? Phytochem Rev 2:87.

2. Billault I, Guiet S, Mabon F, Robins RJ (2001) Natural deuterium distribution in longchain fatty acids is nonstatistical: A site-specific study by quantitative ${ }^{2} \mathrm{H}$ NMR spectroscopy. ChemBioChem 2:425-431.

3. Krull E, Sachse D, Mügler I, Thiele A, Gleixner G (2006) Compound-specific $\delta^{13} \mathrm{C}$ and $\delta^{2} \mathrm{H}$ analyses of plant and soil organic matter: A preliminary assessment of the effects of vegetation change on ecosystem hydrology. Soil Biol Biochem 38:3211-3221.

4. Pond KL, Huang Y, Wang Y, Kulpa CF (2002) Hydrogen isotopic composition of individual $n$-alkanes as an intrinsic tracer for bioremediation and source identification of petroleum contamination. Environ Sci Technol 36:724-728.

5. Sauer PE, Eglinton TI, Hayes JM, Schimmelmann A, Sessions AL (2001) Compound specific $D / H$ ratios of lipid biomarkers from sediments as a proxy for environmental and climatic conditions. Geochim Cosmochim Acta 65:213-222.

6. Huang $Y$, Shuman B, Wang $Y$, Webb T (2002) Hydrogen isotope ratios of palmitic acid in lacustrine sediments record late Quaternary climate variations. Geology 30:1103-1106.

7. Sachse D, Radke J, Gleixner G (2004) Hydrogen isotope ratios of recent lacustrine sedimentary n-alkanes record modern climate variability. Geochim Cosmochim Acta 68:4877-4889.

8. Sessions AL, Sylva SP, Summons RE, Hayes JM (2004) Isotopic exchange of carbon-bound hydrogen over geologic timescales. Geochim Cosmochim Acta 68:1545-1559.

9. Sternberg $L(1988) \mathrm{D} / \mathrm{H}$ ratios of environmental water recorded by $\mathrm{D} / \mathrm{H}$ ratios of plant lipids. Nature 333:59-61.

10. Luo YH, Steinberg L, Suda S, Kumazawa S, Mitsui A (1991) Extremely low D/H ratios of photoproduced hydrogen by cyanobacteria. Plant Cell Physiol 32:897-900.

11. Yakir D, Deniro MJ (1990) Oxygen and hydrogen isotope fractionation during cellulose metabolism in Lemna gibba L. Plant Physiol 93:325-332.

12. Chikaraishi Y, Naraoka H, Poulson SR (2004) Hydrogen and carbon isotopic fractionations of lipid biosynthesis among terrestrial (C3, C4 and CAM) and aquatic plants. Phytochemistry 65:1369-1381.

13. Sessions AL, Burgoyne TW, Schimmelmann A, Hayes JM (1999) Fractionation of hydrogen isotopes in lipid biosynthesis. Org Geochem 30:1193-1200.

14. Chikaraishi Y, Naraoka H, Poulson SR (2004) Carbon and hydrogen isotopic fractionation during lipid biosynthesis in a higher plant (Cryptomeria japonica). Phytochemistry 65:323-330.

15. Chikaraishi Y, Suzuki Y, Naraoka H (2004) Hydrogen isotopic fractionations during desaturation and elongation associated with polyunsaturated fatty acid biosynthesis in marine macroalgae. Phytochemistry 65:2293-2300.

16. Zhang Z, Sachs JP (2007) Hydrogen isotope fractionation in freshwater algae: I. Variations among lipids and species. Org Geochem 38:582-608.

17. Jones A, Sessions AL, Campbell B, Li C, Valentine D (2008) D/H ratios of fatty acids from marine particulate organic matter in the California Borderland Basins. Org Geochem 39:485-500.

18. Li C, Sessions AL, Kinnaman FS, Valentine DL (2009) Hydrogen-isotopic variability in lipids from Santa Barbara Basin sediments. Geochim Cosmochim Acta, 10.1016/ j.gca.2009.05.056

19. Sessions AL, Jahnke LL, Schimmelmann A, Hayes JM (2002) Hydrogen isotope fractionation in lipids of the methane-oxidizing bacterium Methylococcus capsulatus. Geochim Cosmochim Acta 66:3955-3969.

20. Campbell BJ, Li C, Sessions AL, Valentine DL (2009) Hydrogen isotopic fractionation in lipid biosynthesis by $\mathrm{H}_{2}$-consuming Desulfobacterium autotrophicum. Geochim Cosmochim Acta 73:2744-2757.

21. Valentine DL, Sessions AL, Tyler SC, Chidthaisong A (2004) Hydrogen isotope fractionation during $\mathrm{H}_{2} / \mathrm{CO}_{2}$ acetogenesis: Hydrogen utilization efficiency and the origin of lipid-bound hydrogen. Geobiology 2:179-188.

22. Marrakchi H, Zhang Y, Rock CO (2002) Mechanistic diversity and regulation of Type II fatty acid synthesis. Biochem Soc Trans 30:1050-1055.

23. Campbell JW, Cronan JE (2001) Bacterial fatty acid biosynthesis: Targets for antibacterial drug discovery. Annu Rev Microbiol 55:305-332.

24. White SW, Zheng J, Zhang Y, Rock CO (2005) The structural biology of Type II fatty acid biosynthesis. Annu Rev Biochem 74:791-831.

25. Rock CO, Jackowski S (2002) Forty years of bacterial fatty acid synthesis. Biochem Biophys Res Commun 292:1155-1166.

26. Pohlmann A, et al. (2006) Genome sequence of the bioplastic-producing "Knallgas" bacterium Ralstonia eutropha H16. Nat Biotechnol 24:1257-1262.

27. Gottschalk G (1986) Bacterial Metabolism (Springer, New York).

28. White D (2000) The physiology and biochemistry of prokaryotes (Oxford Univ Press, New York).

29. Quayle JR (1961) Metabolism of C1 compounds in autotrophic and heterotrophic microorganisms. Annu Rev Microbiol 15:119-152.

30. Friedrich CG, Bowien B, Friedrich B (1979) Formate and oxalate metabolism in Alcaligenes eutrophus. J Gen Microbiol 115:185-192. organic substrates were analyzed by Dr. A. Schimmelmann (Indiana University, Bloomington) by double-equilibration following the description in SI Methods.

ACKNOWLEDGMENTS. We thank A. Schimmelmann for substrate $\mathrm{D} / \mathrm{H}$ analyses, M. Eek, and L. Zhang for assistance with lipid D/H analyses and J. Leadbetter and D. Newman and their respective research groups for providing insightful discussion and assistance with microbial cultures. This work was supported by National Science Foundation Grant EAR-0645502 (to A.L.S.) and a predoctoral fellowship (to X.Z.).

31. Sessions AL, Hayes JM (2005) Calculation of hydrogen isotopic fractionations in biogeochemical systems. Geochim Cosmochim Acta 69:593-597.

32. Yakir D (1992) Variations in the natural abundance of oxygen-18 and deuterium in plant carbohydrates. Plant Cell Environ 15:1005-1020.

33. Luo YH, Sternberg L (1991) Deuterium heterogeneity in starch and cellulose nitrate of CAM and $C_{3}$ plants. Phytochemistry 30:1095-1098.

34. Sessions AL (2006) Seasonal changes in D/H fractionation accompanying lipid biosynthesis in Spartina alterniflora. Geochim Cosmochim Acta 70:2153-2162.

35. Saito K, Kawaguchi A, Okuda S, Seyama Y, Yamakawa T (1980) Incorporation of hydrogen atoms from deuterated water and stereospecifically deuterium-labeled nicotinamide nucleotides into fatty acids with the Escherichia coli fatty acid synthetase system. Biochim Biophys Acta 618:202-213.

36. Schmidt HL, Werner RA, Eisenreich W (2003) Systematics of ${ }^{2} \mathrm{H}$ patterns in natural compounds and its importance for the elucidation of biosynthetic pathways. Phytochem Rev 2:61-85.

37. Heath RJ, Rock CO (1995) Enoyl-acyl carrier protein reductase (fabl) plays a determinant role in completing cycles of fatty acid elongation in Escherichia coli. J Biol Chem 270:26538-26542.

38. Popjak G (1970) in The Enzymes, ed Boyer PD (Academic, New York), pp 115-215.

39. McMurry JE, Begley TP (2005) The Organic Chemistry of Biological Pathways (Roberts and Company, Englewood, NJ).

40. Simon H, Kraus A (1976) in Isotopes in Organic Chemistry, eds Buncel E, Lee CC (Elsevier Science, Amsterdam), pp 153-229.

41. Ghisla S, Massey V (1989) Mechanisms of flavoprotein catalyzed reactions. Eur J Biochem 181:1-17.

42. Bergler H, Fuchsbichler S, Högenauer G, Turnowsky F (1996) The enoyl-[acyl-carrierprotein] reductase (Fabl) of Escherichia coli, which catalyzes a key regulatory step in fatty acid biosynthesis, accepts NADH and NADPH as cofactors and is inhibited by palmitoyl-CoA. Eur J Biochem 242:689-694.

43. Riley M, et al. (2006) Escherichia coli K-12: A cooperatively developed annotation snapshot-2005. Nucleic Acids Res 34:1-9.

44. Kreuzer-Martin HW, Lott MJ, Ehleringer JR, Hegg EL (2006) Metabolic processes account for the majority of the intracellular water in log-phase Escherichia coli cells as revealed by hydrogen isotopes. Biochemistry 45:13622-13630.

45. Ingraham JL, Maaløe O, Neidhardt FC (1983) Growth of the Bacterial Cell (Sinauer Sunderland, MA).

46. Edens WA, Urbauer JL, Cleland WW (1997) Determination of the chemical mechanism of malic enzyme by isotope effects. Biochemistry 36:1141-1147.

47. Jackson J (2003) Proton translocation by transhydrogenase. FEBS Lett 545:18-24.

48. Shin M (2004) How is ferredoxin-NADP reductase involved in the NADP photoreduction of chloroplasts? Photosynth Res 80:307-313.

49. O'Leary MH (1989) Multiple isotope effects on enzyme-catalyzed reactions. Annu Rev Biochem 59:377-401.

50. Thomson JF, Nance SL, Bush KJ, Szczepanik PA (1966) Isotope and solvent effects of deuterium on aconitase. Arch Biochem Biophys 117:65-74.

51. Jackson JB, Peake SJ, White SA (1999) Structure and mechanism of protontranslocating transhydrogenase. FEBS Lett 464:1-8.

52. Lenz $\mathrm{H}$, et al. (1971) Stereochemistry of si-citrate synthase and ATP-citrate-lyase reactions. Eur J Biochem 24:207-215.

53. Hayes JM (2001) Fractionation of carbon and hydrogen isotopes in biosynthetic processes. Rev Mineral Geochem 43:225-277.

54. Sauer U, Canonaco F, Heri S, Perrenoud A, Fischer E (2004) The soluble and membranebound transhydrogenases UdhA and PntAB have divergent functions in NADPH metabolism of Escherichia coli. J Biol Chem 279:6613-6619.

55. Retey J, et al. (1970) Stereochemical studies of the exchange and abstraction of succinate hydrogen on succinate dehydrogenase. Eur J Biochem 14:232-242.

56. Nesheim JC, Lipscomb JD (1996) Large kinetic isotope effects in methane oxidation catalyzed by methane monooxygenase: Evidence for $\mathrm{C}-\mathrm{H}$ bond cleavage in a reaction cycle intermediate. Biochem 35:10240-10247.

57. Zhao J, Baba T, Mori H, Shimizu K (2004) Effect of zwf gene knockout on the metabolism of Escherichia coli grown on glucose or acetate. Metab Eng 6:164-174.

58. Zhao J, Shimizu K (2003) Metabolic flux analysis of Escherichia coli K12 grown on ${ }^{13} \mathrm{C}$-labeled acetate and glucose using GC-MS and powerful flux calculation method. J Biotechnol 101:101-117.

59. Rodríguez-Ruiz J, Belarbi E, Sánchez JLG, Alonso DL (1998) Rapid simultaneous lipid extraction and transesterification for fatty acid analyses. Biotechnol Tech 12:689-691.

60. Coplen TB (1988) Normalization of oxygen and hydrogen isotope data. Chem Geol 72:293-297.

61. Chikaraishi $Y$ (2003) Compound-specific $\delta D-\delta^{13} C$ analyses of $n$-alkanes extracted from terrestrial and aquatic plants. Phytochemistry 63:361-371. 